\title{
Desempenho na escrita em destros e canhotos em relação ao sexo e à postura
}

\author{
Francisca Morais da Silveira \\ Universidade Federal do Maranhão \\ William Lee Berdel Martin \\ Universidade Federal do Pará
}

\begin{abstract}
RESUMO
A postura invertida para escrever, usada por muitos canhotos, de acordo com a teoria de adaptação técnica, originou-se da necessidade de adaptação às exigências abdutivas implícitas na ortografia romana. No entanto, muitos educadores a julgam prejudicial e, considerando a sua adoção como geradora de problemas físicos e escrita ilegível, não recomendam o uso da postura invertida. Para averiguar a associação da postura invertida a problemas físicos naqueles que a adotam, comparou-se o desempenho gráfico de alunos invertidos e não invertidos. Verificou-se que nenhum problema físico ocorreu em consequiência da adoção desse tipo postura invertida. Em termos de qualidade e velocidade, no desempenho cursivo, as alunas superaram os alunos. Embora os canhotos invertidos tenham apresentado maior lentidão na escrita, o nível de qualidade permaneceu igual aos outros grupos. Os resultados não sustentam a hipótese de que a postura invertida cause problemas físicos ou resulte em escrita inferior e, portanto, não justifica a prática pedagógica de desencorajar a sua adoção pelos alunos canhotos.
\end{abstract}

Palavras-chave: canhotismo; postura para escrever; diferenças de gênero.

\begin{abstract}
Writing performance of right - and left - handers in relation to sex and writing posture

According to the technical adaptation theory, the inverted hand posture used by left-handers arose as an adjustment for coping with abductive demands implicit in Roman orthography. Some educators regarded the posture as maladaptive, leading to physical problems and illegible script, and recommended a non-inverted posture. This study sought to ascertain whether the differential hand posture was associated with physical or pre-natal problems, and compared the writing performance of both inverted and non-inverted writers. It was found that no physical problems stemmed from this inverted posture. On cursive tasks, females outperformed males, and although inverted left-handers tended to write cursive script slower, the level of quality was equal to that of non-inverted sinistrals and dextrals. These results lend no support to the supposition that inversion causes physical problems, or that it leads to inferior writing, and therefore does not justify attempts by educators to dissuade lefthanders from adopting the inverted posture.
\end{abstract}

Keywords: left-handedness; writing posture; sex differences.

No decurso da primeira parte do século 20, alguns pesquisadores observaram que, em relação aos destros, os canhotos apresentam maior variabilidade na postura que adotam para escrever (Gould, 1908; Selzer, 1933). Existem pelo menos três classes de postura manual para escrever: a não invertida (NI), a invertida (I) e uma variante intermediária, às vezes chamada de "semi-invertida" (SI), ou não definida (Peters, 1986; Peters \& Pedersen, 1978). A maioria dos destros escreve na postura NI, posicionando sua mão abaixo da linha de pauta, com a ponta do lápis orientada para cima. Embora muitos canhotos também escrevam com a postura NI, uma percentagem substancial adota a postura invertida, dobrando a mão acima da linha de pauta, com a ponta do lápis orientada na direção do corpo. Os sujeitos que adotam a postura SI tendem a escrever com a mão paralela à linha de pauta, e a ponta do lápis apontando na direção horizontal. Segundo um levantamento realizado por Paixão (2002), entre alunos destros paraenses, cerca de $91 \%$ escreveram com a postura NI, 6\% na postura I e 3\% na posição SI. Entre os canhotos, $55 \%$ preferiram a postura I, $38 \%$ a NI, e os demais $8 \%$ escreveram com a postura SI.

A postura I, sendo pouco freqüente em destros, pode ser atribuída a fatores aleatórios, onde a inversão consiste num hábito adquirido por acaso e mantido por costume e condicionamento. Entre canhotos, onde o traço ocorre com maior freqüência, de modo geral, as explicações quanto à origem tendem a ser resumidas em duas classes de teoria: patologia ou adaptação técnica.

Com referência à primeira, a posição mais radical defendida por Bakan $(1975,1990)$, afirma que todas as expressões fenotípicas de canhotismo decorrem de patologia no sistema nervoso central, ocasionada por 
complicações no parto. Enquanto Coren (1992) e Coren e Halpern (1991) sugeriram que em pelo menos a metade da população canhota, certos traços, como a postura invertida, são sintomas de neuropatologia. Este postulado surgiu de um levantamento conduzido por Searleman, Porac e Coren (1982). Eles encontraram uma incidência maior de problemas no parto (i.e. hipoxia, parto prematuro, incompatibilidade sanguiínea e baixo peso, entre outros) nos canhotos invertidos do que nos NIs. Este modelo patológico tem sido duramente criticado por apresentar falhas metodológicas e fazer generalizações exageradas com base em evidências fracas (Harris, 1993; Harris \& Carlson, 1988; Martin \& Freitas, 2002; Schwartz, 1990). Outros estudos não encontraram nenhuma associação entre condições neuropatológicas e canhotismo (McKeever, Suter \& Rich, 1995; Schwartz, 1990) e nem entre a incidência de problemas no parto e postura manual entre canhotos (Levander \& Schalling, 1988).

Por outro lado, a teoria de adaptação social tem maior aceitação entre pesquisadores na área de lateralidade. Selzer (1933) achava que a postura diferencial estava relacionada à configuração de movimentos físico-musculares, envolvidos na produção de grafia. Nestes termos, nos sistemas ortográficos baseados na convenção romana, a direção de escrever prossegue da esquerda para a direita da página e acarreta dificuldades aos canhotos. As dificuldades surgem porque essa direção é vista como contrária à "tendência inata" do canhoto para escrever da direita para a esquerda (Burt, 1937; Hildreth, 1950; Reed \& Smith, 1961). Há muito tempo pedagogos e psicólogos atribuíram um maior grau de eficiência na escrita ao movimento abdutivo em relação ao adutivo. No primeiro, o lápis é puxado através da página, afastando-se da linha média do corpo; enquanto no segundo, o lápis é "empurrado" através da folha na direção do corpo. O movimento abdutivo é característico dos destros (NIs e Is) e o segundo dos canhotos NIs. Neste último grupo, a progressão adutiva tende a obstruir a retroalimentação visual porque a mão esquerda esconde o que já foi escrito, resultando, muitas vezes em erros de soletração. Para evitar tais problemas, muitos canhotos NIs inclinam o papel para a direita em ângulos variando entre 45 a 90 graus (Hildreth, 1950; Ure, 1969).

Desta perspectiva, a postura invertida, adotada por canhotos, é vista como uma outra solução técnica, numa tentativa de fazer as adaptações físicas necessárias para enfrentar as exigências de escrita cursiva numa convenção ortográfica contralateral à orientação natural deles. Usando essa postura, o canhoto consegue escrever abdutivamente e, desde que posicione o punho acima da linha de pauta, não encontra nenhum impedimento visual nem problemas de borrar a tinta (Clark, 1974; Herron, 1980; Meulenbroeck \& Van Galen, 1989; Shepherd, 1986).

Existe um paradoxo: muitos especialistas, interessados em retificar os problemas enfrentados por alunos canhotos, desaconselham o uso da postura invertida, quando aprendem a escrever na forma cursiva, embora aceitem a premissa básica da teoria de adaptação. Clark (1974, p. 33) asseverou que "essa postura não é normal”. Cole (1939), Enstrom (1962) e Hildreth (1950) argumentaram que a postura invertida produz problemas físicos que podem prejudicar a saúde. Vários especialistas enfatizaram que a postura invertida aumenta a tensão muscular, causa fadiga, cãibras, problemas de coluna, e resulta em escrita lenta e/ou ilegível. (Clark, 1974; Cole, 1939; Fagg, 1989ab; Langford, 1984; Martin, 1952). Machado (1982), numa brochura publicada pela Associação Brasileira para Canhotos, ressaltou que: "essa postura deve ser corrigida porque, além de cansativa, pode causar deformação na coluna" (p. 43). Fagg (1989b) reforçou esse conselho em um livreto. A autora apresentou duas fotografias de uma canhota escrevendo nas duas posturas: a fotografia da postura NI leva o rótulo de "CORRETA", enquanto que a fotografia mostrando a postura invertida foi rotulada como "ERRADA" (p. 2). Num livro preparado para alunos canhotos, Congdon (1989) mostrou desenhos de cada postura. $\mathrm{O}$ desenho da postura NI foi rotulado como "BOM" e a invertida como "MAU" (p. 10). Todos estes especialistas em caligrafia recomendaram que os canhotos sejam instruídos na postura NI, com a folha de escrever "inclinada para a direita em um ângulo de 45 graus" (Machado, 1982, p. 42).

Em certos casos, há evidência direta de que a última recomendação foi posta em prática (cf. Peters, 1986). Por exemplo, como parte de seu experimento, Szeligo, Brazier e Houston (2003) obtiveram informações detalhadas dos respondentes canhotos. Estes pesquisadores descobriram que, enquanto cerca de $50 \%$ dos canhotos invertidos sofreram tentativas por parte das professoras visando mudar sua postura para a posição NI, nenhum dos NIs foi instruído a adotar a postura invertida.

A questão da validade de algumas das suposições, citadas acima, incentivou esta pesquisa. Inicialmente, nos parecia que as declarações quanto aos riscos da postura invertida para a saúde consistem em impressões subjetivas, pois não encontramos nenhuma evidência empírica que as sustentem. Posteriormente, verificamos que poucas investigações foram planejadas para comparar a qualidade e a velocidade da escrita entre destros e canhotos invertidos e não invertidos, e nas realizadas, os resultados foram contraditórios.

Independentemente da postura, dois estudos relataram que, em termos da qualidade do produto escrito, o nível de desempenho era mais alto nos destros do que nos canhotos (Lauterbach, 1933; Suen, 1983). Outras três investigações não encontraram nenhuma diferença entre destros e canhotos, ou na qualidade ou na velocidade da escrita (Groff, 1964; Pyle \& Drouin, 1932; Smith \& Reed, 1959). Quando a postura passou a ser considerada, Enstrom (1962) aplicou uma escala padronizada de proficiência na escrita e, em relação aos canhotos invertidos, os NIs obtiveram os escores mais altos. Dois experimentos avaliaram a legibilidade e a velocidade na escrita e não identificaram nenhuma divergência entre $o$ desempenho dos invertidos e NIs (Athenes \& Guiard, 1991; Peters \& McGrory, 1987). Dessas duas últimas 
investigações, a de Peters e McGrory (1987) foi mais detalhada, porque, além dos canhotos Is e NIs, eles conseguiram incluir uma amostra de destros invertidos. Por outro lado, as tarefas foram limitadas a apenas uma frase cursiva, muito simples, e à escrita da letra cursiva "ele" repetidas vezes (i.e. "llll"), assim, talvez os estímulos fossem muito fáceis para identificar quaisquer diferenças verdadeiras entre os grupos na qualidade e velocidade. Levando em conta as considerações acima, este experimento teve os seguintes objetivos: (1) averiguar se, de fato, a postura invertida, em particular em canhotos, está relacionada com problemas físicos; (2) averiguar se a inversão está associada com antecedentes neuropatológicos, decorrentes de complicações no parto; (3) verificar se existe uma relação funcional entre preferência e postura manual e a qualidade e velocidade na grafia, cursiva e não cursiva; e (4) identificar quaisquer outras variáveis, tais como sexo e grau de consistência que estejam associadas ao nível de desempenho. Seguimos em parte o roteiro de Peters e McGrory (1987), ampliamos a quantidade de estímulo escrito e estendemos a avaliação para frases não cursivas.

\section{MÉTODO}

\section{Amostras}

Foram selecionados 96 adolescentes, 48 destros e 48 canhotos entre a idade de 14 a 18 anos $(\mathrm{M}=15,84$, $\mathrm{DP}=1,25)$ de quatro escolas públicas do ensino médio localizadas em dois bairros de São Luiz, Maranhão. Essas escolas atendem alunos oriundos de famílias de baixa renda. Deste total, classificamos 24 alunos em cada uma das quatro classes de postura: destros NIs, destros invertidos, canhotos NIs e canhotos invertidos. Estes subgrupos foram divididos igualmente entre os sexos. A participação era voluntária e os que aceitaram o convite foram remunerados.

\section{Medidas e Materiais \\ O Inventário de Preferências Laterais (IPL)}

O IPL foi desenvolvido por Martin (1990) com a finalidade de medir a direção e o grau de preferência manual (PM) e podálica. Áqui descrevemos apenas o teste de PM. Além da classificação nominal (destro e canhoto), o IPL é composto de 10 tarefas manuais: escrever, pentear o cabelo, desenhar, martelar, recortar com tesouras, riscar um fósforo, arremessar uma bola, descartar baralhos, enroscar uma porca em pino e serrar. O respondente é avaliado, em cada item, através de uma escala de cinco pontos: $+2=$ mão direita, sempre; $+1=$ mão direita, usualmente; $0=$ ambas, igualmente; -1 = mão esquerda, usualmente e 2 = mão esquerda, sempre. A soma total resulta num escore manual (EM) que varia entre +20 e -20 pontos. Se a direção da grafia e o EM concordasse com a atribuição nominal, o indivíduo seria classificado como destro ou canhoto, e todos os participantes escreveram com a mão dominante.

\section{Avaliação da Postura para Escrever}

Os desenhos representativos das posturas diferenciadas aparecem na última folha do IPL. Além de autoclassificação, cada aluno escreveu seu nome numa linha horizontal dentro de um desenho de um relógio e o experimentador avaliou a postura com base no critério adotado de Levy (1984). Se a mão fosse posicionada acima da linha de pauta e a ponta do lápis consistentemente orientada para a parte inferior do relógio (entre 4:00 a 8:00 horas), o aluno era classificado como invertido. Se a mão fosse colocada abaixo da linha e a ponta do lápis apontando para acima (entre 22:00 e 2:00 horas), o aluno era classificado como NI. Os semi-invertidos e os casos duvidosos foram excluídos.

\section{Estímulo Escrito: Frases e Letras}

No caso das frases, cinco provérbios foram lidos pelo experimentador na seguinte ordem: (Treino) "Casa de ferreiro, espeto de pau"; (1) "Mais vale um pássaro na mão do que dois voando"; (2) "Ladrão que rouba de ladrão tem cem anos de perdão"; (3) "Água mole em pedra dura, tanto bate até que fura": (4) "Quanto mais alto o coqueiro, maior o tombo". Após a leitura, o aluno escrevia a frase, primeiro em letra de forma e depois em letra cursiva, acima de linhas de $20 \mathrm{~cm}$ de comprimento impressas no caderno de respostas. As frases eram lidas tantas vezes quanto necessárias ao seu perfeito entendimento. Posteriormente foram apresentadas quatro letras cursivas, escritas em uma cartolina (tamanho $30 \mathrm{~cm}$ x $15 \mathrm{~cm}$ ): (treino) "aaa"; (1) "lll"; (2) "ooo"; (3) "zzz". O experimentador demonstrava como escrevê-las; após, solicitava ao aluno que fizesse o mesmo até preencher as linhas de $20 \mathrm{~cm}$, existentes no caderno de respostas.

\section{O Inventário Sociomédico (ISM)}

O ISM foi preenchido junto com as mães ou os responsáveis pelos alunos e teve como objetivo obter dados sobre a ocorrência de possíveis complicações no parto. Segundo o esquema de Searleman e cols. (1982), uma combinação das seguintes condições foi julgada como "indicadora" de neuropatologia: parto demorado ou prematuro, hipoxia, cianose, incompatibilidade sangüínea, convulsões e golpes envolvendo o crânio.

\section{Procedimento}

Cada aluno foi avaliado individualmente em todas as medidas apresentadas, na ordem indicada acima. Enquanto o aluno escrevia as frases e letras, o tempo de resposta (TR) foi cronometrado (em 1/10 de segundos), por tentativa. O resultado foi somado e dividido pelo número total de tentativas. Duas professoras, do curso de letras da UFMA, julgaram a qualidade caligráfica de cada frase cursiva, não cursiva e de cada bloco de letras cursivas, em uma escala ordinal de um (1) a cinco (5) pontos; onde: $1=$ desempenho muito inferior; 2 = desempenho inferior; 3 = desempenho médio; $4=$ desempenho bom e $5=$ 
desempenho muito bom. Na atribuição desses valores, as professoras usaram critério referente aos aspectos caligráficos: inteligibilidade das letras e palavras, uniformidade de espaço entre letras e palavras, legibilidade na passagem de uma letra para outra, alinhamento do eixo das letras, alinhamento paralelo das letras e palavras na linha de pauta, simetria e consistência na formação das letras e palavras, e consistência na inclinação da escrita. O grau de concordância entre as julgadoras foi analisado através do coeficiente de correlação de Spearman $\left(r_{s}\right)$. Em todos as três classes de respostas a concordância era alta: $r_{\mathrm{s}}(96)=0,93$ para as frases cursivas, $r_{\mathrm{s}}(96)=0,88$ para as frases não cursivas e $r_{s}$ $(96)=0,90$ para as letras cursivas.

\section{Análises Estatísticas}

A maior parte das análises envolve o nível de desempenho nas frases e nas letras. As variáveis dependentes (VDs) foram: a qualidade e o tempo gasto para escrever o estímulo (TR). No início houve seis VIs possíveis. Após o exame do resultado das análises de regressão múltipla (ARM), usando o procedimento seqüencial, encontramos três VIs associadas significativamente com pelo menos duas das VDs: sexo, preferência e postura manual. Depois foi aplicada a ANOVA fatorial para o plano de amostras independentes, e quando os efeitos principais e/ou as interações foram significativos $(\mathrm{p}<0,05)$, aplicamos o teste HSD de Tukey para identificar quais as médias que diferiram entre si.

\section{RESULTADOS}

\section{Problemas Físicos e Congênitos}

Na primeira análise avaliamos a afirmação quanto aos supostos problemas físicos causados nos canhotos, pela postura invertida. Dos 96 alunos, apenas cinco canhotos, três invertidos e dois NIs, indicaram através do IPL a presença de problemas físicos. Segundo os alunos, esses problemas ocorreram por falta de carteiras escolares adaptadas e não relacionados à postura adotada para escrever.

$\mathrm{Na}$ segunda série de análises, referente à freqüência de complicações no parto, as comparações entre os grupos foram efetuadas por meio do "odds ratio" (OR), com o intervalo de confiança ao nível de 95\% ( $\left.\mathrm{IC}_{0,95}\right)$ dado entre parênteses, e o valor de alfa (p) obtido do teste exato de Fisher. Na amostra total, a incidência de antecedentes neuropatológicos foi bastante alta, aproximadamente $45 \%$. Houve pouca diferença entre os destros e canhotos (46\% vs. 44\%). $\mathrm{Na}$ avaliação da postura, uma diferença significativa foi encontrada. As complicações ocorreram mais freqüentemente nos destros NIs do que nos destros invertidos (63\% vs. $29 \%)$ : $\mathrm{OR}=4,05\left(\mathrm{IC}_{0,95}=1,21-\right.$ $13,54), p=0,042$, indicando que o primeiro grupo era quatro vezes mais provável de estar em alto risco do que o segundo grupo. Embora os canhotos NIs tivessem uma maior incidência de problemas associados ao parto do que os invertidos (54\% vs. $33 \%$ ), a diferença não era significativa. Quando combinamos os dados, considerando a inversão e a não inversão para escrever, independentemente da $\mathrm{PM}$, verificamos que $58 \%$ dos alunos com postura NI evidenciaram complicações, em comparação com $31 \%$ dos invertidos; uma diferença significativa: $\mathrm{OR}=$ $3,08\left(\mathrm{IC}_{0,95}=1,33-7,12\right), \mathrm{p}=0,013$, indicando que a probabilidade de apresentar antecedentes neuropatológicos entre os NIs era três vezes superior à dos invertidos.

\section{O Nível de Qualidade e Velocidade nas Frases e Letras}

As três tabelas seguintes resumem os valores das médias e dos desvios padrões para cada tarefa através dos grupos e subgrupos principais. Não apresentamos os dados para os oito subgrupos menores onde o sexo foi cruzado com a postura (e.g. os destros NIs, destras NIs e assim por diante). Na presença de efeitos interativos e quando o teste de Tukey identificou diferenças significativas entre essas subamostras, o valor de cada média foi citado entre parênteses.

Tabela 1: A Qualidade e Velocidade Média das Frases Cursivas em Função do Sexo, Preferência Manual e Postura para Escrever

\begin{tabular}{lccccc}
\hline \multicolumn{1}{c}{ Grupos } & Nualidade & \multicolumn{2}{c}{ Velocidade } \\
& N & M & DP & M & DP \\
\hline Sexo & & & & & \\
Masculino & 48 & 4,30 & 0,47 & 119,67 & 25,16 \\
Feminino & 48 & 4,61 & 0,42 & 107,02 & 30.03 \\
Preferência Manual & & & & & \\
Destro & 48 & 4,59 & 0,44 & 108,73 & 9,24 \\
Canhoto & 48 & 4,31 & 0,47 & 117,96 & 26,79 \\
Preferência Manual por Sexo & & & & & \\
Destros & 24 & 4,40 & 0,44 & 117,33 & 23,19 \\
Destras & 24 & 4,78 & 0,36 & 100,13 & 32,48 \\
Canhotos & 24 & 4,19 & 0,48 & 122,00 & 27,28 \\
Canhotas & 24 & 4,44 & 0,43 & 113,92 & 26,24 \\
Postura para Escrever & & & & & \\
Destro Não Invertido & 24 & 4,61 & 0,37 & 105,17 & 31,20 \\
Destro Invertido & 24 & 4,57 & 0,50 & 112,29 & 27,34 \\
Canhoto Não Invertido & 24 & 4,25 & 0,49 & 108,88 & 27,43 \\
Canhoto Invertido & 24 & 4,38 & 0,45 & 127,04 & 23,29 \\
\hline
\end{tabular}


No que se refere ao nível de qualidade das frases cursivas, a ANOVA fatorial 2 (sexo) x 2 (PM) x 2 (postura) foi aplicada aos dados resumidos na Tabela 1. Foram encontrados efeitos principais para sexo, $\mathrm{F}$ $(1,88)=13,89, \mathrm{p}<0,001$, e uma interação entre sexo e postura, $\mathrm{F}(1,88)=8,57, \mathrm{p}<0,004$. O desempenho das alunas em geral foi julgado superior ao dos alunos $(\mathrm{M}=4,61$ vs. $\mathrm{M}=4,30)$ e a interação decorreu do alto nível de qualidade atribuída às destras invertidas, que diferiram significativamente dos destros e canhotos invertidos e dos canhotos NIs ( $\mathrm{M}=4,96$ vs. $\mathrm{M}=4,18$,
$\mathrm{M}=4,21$ e $\mathrm{M}=4,17:$ teste de Tukey, $\mathrm{p}<0,008)$, respectivamente.

Em termos da velocidade em que as frases cursivas foram escritas, a ANOVA fatorial 2 (sexo) x 2 (PM) x 2 (postura) revelou dois efeitos principais: um para o fator sexo, $\mathrm{F}(1,88)=5,23, \mathrm{p}<0,03$, e outro para postura, $\mathrm{F}(1,88)=5,20, \mathrm{p}<0,03$. As alunas escreveram as frases com mais rapidez que os alunos $(\mathrm{M}=107,02$ vs. $\mathrm{M}=119,7)$ e em comparação com os destros NIs, os canhotos invertidos escreveram as frases mais lentamente $(M=127,04$ vs. $M=105,2$ : teste de Tukey, $\mathrm{p}<0,02)$.

Tabela 2: A Qualidade e Velocidade Média das Frases em Letra de Forma em Função do Sexo, Preferência Manual e Postura para Escrever

\begin{tabular}{lccccc}
\multicolumn{1}{c}{ Grupos } & Nualidade & \multicolumn{3}{c}{ Velocidade } \\
& N & M & DP & M & DP \\
\hline Sexo & 48 & 4,23 & 0,55 & 164,25 & 62,77 \\
Masculino & 48 & 4,25 & 0,73 & 153,75 & 46,37 \\
$\begin{array}{l}\text { Feminino } \\
\text { Preferência Manual }\end{array}$ & & & & & \\
Destro & 48 & 4,41 & 0,67 & 152,52 & 55,72 \\
Canhoto & 48 & 4,07 & 0,58 & 165,48 & 54,38 \\
Preferência Manual por Sexo & & & & & \\
Destros & 24 & 4,38 & 0,58 & 158,38 & 60,31 \\
Destras & 24 & 4,44 & 0,76 & 146,67 & 51,33 \\
Canhotos & 24 & 4,08 & 0,48 & 170,13 & 65,90 \\
Canhotas & 24 & 4,06 & 0,66 & 160,83 & 40,68 \\
Postura para Escrever & & & & & \\
Destro Não Invertido & 24 & 4,50 & 0,78 & 143,96 & 56,14 \\
Destro Invertido & 24 & 4,31 & 0,53 & 161,08 & 55,12 \\
Canhoto Não Invertido & 24 & 4,06 & 0,52 & 158,83 & 62,55 \\
Canhoto Invertido & 24 & 4,08 & 0,64 & 172,13 & 45,43 \\
\hline
\end{tabular}

Os dados referentes ao julgamento da qualidade das frases escritas em letra de forma aparecem na Tabela 2. Com base nas mesmas VIs (sexo, PM e postura), apenas um efeito emergiu para o fator PM: $\mathrm{F}(1,88)=6,75, \mathrm{p}<0,02$. Este resultado reflete a maior qualidade da caligrafia dos destros em relação à dos canhotos $(\mathbf{M}=4,41$ vs. $\mathbf{M}=4,07)$. Em termos qualitativos, embora a grafia tenha sido legível, os canhotos tenderam a persistir na escrita cursiva quando tentaram formar as letras separadamente. No que se refere à velocidade, nenhuma diferença foi estatisticamente significativa.

Tabela 3: A Qualidade e Velocidade Média das Letras Cursivas em Função do Sexo, Preferência Manual e Postura para Escrever

\begin{tabular}{lccccc} 
& \multicolumn{3}{c}{ Qualidade } & \multicolumn{3}{c}{ Velocidade } \\
\multicolumn{1}{c}{ Grupos } & N & M & DP & M & DP \\
\hline Sexo & & & & & \\
Masculino & 48 & 4,36 & 0,45 & 63,19 & 20,96 \\
Feminino & 48 & 4,57 & 0,54 & 58,31 & 20,32 \\
$\begin{array}{l}\text { Preferência Manual } \\
\text { Destro }\end{array}$ & & & & & \\
Canhoto & 48 & 4,44 & 0,52 & 60,02 & 21,01 \\
Preferência Manual por Sexo & 48 & 4,49 & 0,51 & 61,48 & 20,54 \\
Destros & & & & & \\
Destras & 24 & 4,31 & 0,52 & 62,71 & 21,30 \\
Canhotos & 24 & 4,58 & 0,49 & 57,33 & 20,82 \\
Canhotas & 24 & 4,41 & 0,38 & 63,61 & 21,06 \\
Postura para Escrever & 24 & 4,57 & 0,61 & 59,29 & 20,22 \\
Destro Não Invertido & & & & & \\
Destro Invertido & 24 & 4,61 & 0,37 & 61,71 & 19,79 \\
Canhoto Não Invertido & 24 & 4,28 & 0,59 & 58,33 & 22,46 \\
& 24 & 4,54 & 0,44 & 57,79 & 18,91
\end{tabular}


Em relação à qualidade média das letras interconectas, a ANOVA fatorial acusou efeitos significativos para sexo, $\mathrm{F}(1,88)=4,64, \mathrm{p}<0,04$, postura, $\mathrm{F}(1,88)=5,00, \mathrm{p}<0,03$ e uma interação entre sexo e postura, $\mathrm{F}(1,88)=4,63, \mathrm{p}<0,04$. Em termos gerais, a qualidade do desempenho das alunas foi maior do que a dos alunos $(M=4,57$ vs. $M=4,36)$ e, em contraste com os destros NIs, os destros invertidos realizaram um desempenho inferior $(\mathrm{M}=$ 4,61 vs. $\mathrm{M}=4,28$ : teste de Tukey, $\mathrm{p}<0,05$ ). Mais especificamente, uma avaliação das subamostras (postura por sexo) revelou que o menor nível de qualidade atribuído aos destros invertidos, os diferenciou das destras e canhotas NIs ( $\mathrm{M}=3,99$ vs. $\mathrm{M}=4,63$ e $\mathrm{M}=4,68$ : teste de Tukey, $\mathrm{p}<0,05)$.

Em relação à velocidade, uma interação significativa foi encontrada entre sexo e postura, F (1, $88)=5,58, p<0,03$. Este resultado reflete a diferença entre dois subgrupos de canhotos do sexo masculino: os não invertidos escreveram as letras com mais rapidez do que os invertidos ( $\mathrm{M}=51,5$ vs. $\mathrm{M}=75,8$ : teste de Tukey, $\mathrm{p}<0,05)$.

\section{DISCUSSÃO E CONCLUSÃO}

A suposição apresentada por alguns especialistas em caligrafia, além de alguns psicólogos e educadores, de que a postura invertida é causadora de perturbações físicas, com base em nossos dados, não foi sustentada. Nenhum dos destros invertidos queixou-se de tais problemas e os poucos canhotos que os citaram, atribuíram-nos ao uso de carteiras construídas para destros. Não se identifica a origem do argumento que defende a suposta ocorrência de problemas físico-musculares em canhotos invertidos. Há apenas alguns estudos de casos que produziram conclusões subjetivas (cf. Burt, 1937; Hildreth, 1950) que, aparentemente, sem nenhum fundamento, acabaram sendo aceitas de forma não crítica e repetidas por alguns autores subsequientes (e.g. Congdon, 1989; Fagg, 1989ab; Machado, 1982).

Com relação à teoria de canhotismo patológico, nossos resultados não deram respaldo às especulações de Coren (1992) de que o canhotismo constitui um "indicador" de trauma cerebral. Em concordância com os achados de McKeever e cols. (1995) e Schwartz (1990), como mostramos, a incidência de complicações na fase pré e pós-natal ocorreu com a mesma freqüência em destros e canhotos. Houve ainda uma freqüência de complicações significativamente inferior nos alunos destros e canhotos com postura invertida em relação à taxa elevada encontrada nos alunos destros e canhotos com postura não invertidos. Tais resultados contradizem os dados apresentados

por Searleman e cols. (1982) e de modo geral, vão de encontro aos achados relatados por Levander e Schalling (1988) para canhotos invertidos e não invertidos, com uma diferença: estes últimos pesquisadores não encontraram nenhuma divergência significativa na prevalência de trauma no parto entre os invertidos e os NIs. No presente estudo constatouse um percentual elevado (45\%) de antecedentes neuropatológicos. Convém lembrar, que nossas amostras originam-se de famílias carentes, com altos índices de doenças contagiosas, desnutrição infantil e materna, e precário atendimento para gestantes. A alta prevalência de problemas no parto reflete esta realidade social.

Considerando-se a qualidade e a velocidade, o resultado mais consistente refere-se ao melhor desempenho realizado pelas alunas do que pelos alunos. Estes dados dão apoio adicional à evidência de uma superioridade, no aspecto da grafia, por parte do sexo feminino (Blöte \& Hanstra-Bletz, 1991; Groff, 1964; Peters \& McGrory, 1987). Quanto à velocidade, normalmente não se encontra diferença clara entre os sexos (Athenes \& Guiard, 1991; Peters \& McGrory, 1987; Smith \& Reed, 1959). Obtivemos dados que concordam com os achados do estudo de Ziviani (1984), revelando que, a partir de 12 anos, as alunas escrevem mais rapidamente do que os alunos.

Nas frases e letras cursivas, o desempenho dos canhotos, como grupo, situou-se no mesmo nível de qualidade apresentado pelos destros, um achado que está de acordo com os dados relatados por outras investigações (Groff, 1964; Peters \& McGrory, 1987; Pyle \& Drouin, 1932). A única divergência ocorreu com as frases em letras de forma, onde a produção dos canhotos foi, em média, inferior à dos destros. Os canhotos, mais do que os destros, tenderam a escrever as frases de forma "semi-cursiva". É possível que tal tendência reflita um problema "rítmico", relacionado aos movimentos adutivos feitos por canhotos quando manipulam o lápis através da página. Isto é, uma vez condicionados a escrever cursivamente, alguns apresentam dificuldades, modificando o ritmo dos movimentos, quando obrigados a mudar e escrever o estímulo nas configurações em letras de forma. Por outro lado, a redução na qualidade demonstrada pelos canhotos não deve ser vista como um problema ao longo prazo. Na redação, a partir da sexta série, os professores dão mais ênfase à aptidão na escrita cursiva.

Não encontramos nenhuma evidência em prol da suposição de que canhotos invertidos escrevem com menos precisão e legibilidade do que os não invertidos. O único padrão de desempenho, quase consistente, ocorreu com a velocidade. Os canhotos invertidos escreveram o estímulo cursivo com mais lentidão do que os destros não invertidos. Existe a possibilidade do TR lento decorrer da dificuldade em escrever em carteiras fabricadas para destros. Segundo Herron (1980), quando o canhoto adota a posição invertida os graus de liberdade nos movimentos dos flexores e tensores são reduzidos. Escrever numa carteira desenvolvida para destros implica numa série de contorções corporais por parte dos invertidos. Assim, o TR mais lento pode consistir numa estratégia, na qual, com os graus de liberdade 
reduzidos nos movimentos digitais, talvez os invertidos priorizem a exatidão e qualidade, em detrimento da rapidez nas respostas. Observamos que enquanto os canhotos NIs também apresentam dificuldades escrevendo nessas carteiras, eles tendem a ajustar o ângulo da folha de escrever e conseguem superando, em parte, essa restrição manual.

Essa hipótese alternativa aponta para uma limitação que compromete a validade externa deste experimento. Sendo composto unicamente de alunos de famílias de baixa renda, as amostras não podem ser consideradas como representativas da população estudantil geral ao nível do ensino médio. As escolas públicas localizadas nos bairros periféricos de São Luiz apresentam diversas carências estruturais e certamente não possuem recursos suficientes para atender necessidades específicas dos alunos canhotos. Por este motivo, este estudo deve ser replicado na íntegra, com alunos de famílias de renda média e acima, de preferência lotada em escolas particulares que tenham no mínimo, carteiras sem tendência para a direita ou para a esquerda. Assim, seria possível avaliar a generalidade destes resultados obtidos com alunos carentes.

Em resumo, com base em nossos dados, a hipótese do "invertido desajeitado" não foi sustentada. Segundo a ARM, a proporção maior da variância no desempenho foi atribuída ao sexo, e uma pequena fração foi associada com a preferência e a postura manual. Em termos de legibilidade e qualidade, o desempenho cursivo dos canhotos invertidos não foi inferior ao dos demais grupos. Estes achados minam a validade do estereótipo de anormalidade atribuído aos alunos canhotos que preferem a posição invertida. Nossa conclusão é a de que não existe nenhuma justificativa para proibir o uso dessa postura em alunos canhotos, mas isto não significa que eles devam ser abandonados. Quando sentirem alguma dificuldade na escrita, medidas específicas devem ser adotadas para auxiliá-los. Prevemos, que tais dificuldades venham a ocorrer mais frequientemente em função de certos arranjos nos ambientes físicos, desenvolvidos em favor da maioria destra, do que a postura para escrever.

\section{Referências}

Athenes, S. \& Guiard, Y. (1991). Is the inverted posture really so bad for left-handers? Em J. Wann, A. Wing, \& N. Slõvik (Orgs.), Development of graphic skills (pp. 137-149). London: Academic Press.

Bakan, P. (1975). Are left-handers brain damaged? New Scientist, 67, 200-2002.

Bakan, P. (1990). NonRight-handedness and the continuum of reproductive casuality. Em S. Coren (Org.), Left-handedness: Behavioural implications and anomalies (pp. 33-74). Amsterdam: Elsevier.

Blöte, A. \& Hanstra-Bletz, I. (1991). A longitudinal study on the structure of handwriting. Perceptual and Motor Skills, 72, 983-994.

Burt, C. (1937). The backward child. New York: McMillan.
Clark, M. M. (1974). Teaching left-handed children (2nd Ed.). London: Hodder \& Stoughton Educational.

Cole, L. (1939). Instruction in penmanship for the left-handed child. Elementary School Journal, 39, 436-448.

Congdon, P. J. (1989). Lefty: A story of left-handedness. London: GCIC Publications.

Coren, S. (1992). The left-hander syndrome: The causes and consequences of left-handedness. NY: Free Press.

Coren, S. \& Halpern, D. (1991). Left-handedness: A marker for decreased survival fitness. Psychological Bulletin, 109, 90106.

Enstrom, E. A. (1962). The relative efficiency of various approaches to writing with the left hand. Journal of Educational Research, 55, 283-298.

Fagg, R. (1989a). Helping your left-handed child. London: W. H. Smith.

Fagg, R. (1989b). Helping left-handed children to enjoy handwriting. London: W.H. Smith.

Gould, G. M. (1908). Right-handedness and left-handedness with chapters treating of the writing posture, rules of the road, etc. Philadelphia: J.P. Lippencott.

Groff, P. J. (1964). Who are the better writers-the left-handed or the right-handed? Elementary School Journal, 65, 92-96.

Harris, L. J. (1993). Do left-handers die sooner than righthanders? Comentary on Coren and Halpern's "Lefthandedness: A marker for decreased survival fitness". Psychological Bulletin, 114, 203-234.

Harris, L. J. \& Carlson, D. F. (1988). Pathological lefthandedness: An analysis of theories and evidence. Em D. L. Molfese \& S.J. Segalowitz (Orgs.), Brain lateralization in children (pp. 289-372). NY: Guilford.

Herron, J. (1980). Two hands, two brains, two sexes. Em J. Herron (Org.), Neuropsychology of left-handedness (pp. 233260). NY: Academic Press.

Hildreth, G. (1950). The development and training of hand dominance: V. Training of handedness. Journal of Genetic Psychology, 76, 101-140.

Langford, S. (1984). The left-handed book: How to get by in a right-handed world. London: Grafton.

Lauterbach, C.E. (1933). Shall the left-handed child be converted? Journal of Genetic Psychology, 43, 454-462.

Levander, M. \& Schalling, M. (1988). Self-assessed and examiner-assessed writing posture in Swedish left-handers. Neuropsychologia, 26, 777-781.

Levy, J. (1984). Review, analysis, and some new data on hand posture distributions in left-handers. Brain and Cognition, 3, $105-127$.

Machado, D.V.M. (1982). Meu filho é canhoto: O que saber e como agir em relação ao canhotismo. São Paulo: ALMED.

Martin, K.L. (1952). Handedness: A review of the literature on the history, development and research of laterality preference. Journal of Educational Research, 45, 527-533.

Martin, W.L.B. (1990). Assimetrias direitas-esquerdas no comprimento da mão e do pé em relação ao sexo, preferência manual e podálica. Cadernos do Centro de Filosofia $e$ Ciências Humanas da UFPa., no. 21, 1-29. 
Martin, W. L. B. \& Freitas, M.B. (2002). Mean mortality among Brazilian left- and right-handers: Modification or selective elimination? Laterality, 7, 31-44.

McKeever, W.F.; Suter, P.J. \& Rich, D.A. (1995). Maternal age and parity correlates of handedness: Gender, but no parental handedness modulation of effects. Cortex, 25, 581-589.

Meulenbroeck, R. G. P. \& Van Galen, G. P. (1989). Variations in cursive handwriting as a function of handedness, hand posture and gender. Journal of Human Movement Studies, 16, 239254.

Paixão, C. B. P. (2002). Os efeitos de idade sobre o padrão de preferência manual, podálica e desempenho manual em destros e canhotos. Dissertação de Mestrado não publicada, Universidade Federal do Pará. Belém, PA.

Peters, M. (1986). Incidence of the inverted writing position in a sample of 2194 German elementary school children. Neuropsychologia, 24, 429-433.

Peters, M. \& McGrory, J. (1987). The writing performance of inverted and noninverted right- and left-handers. Canadian Journal of Psychology, 41, 20-32.

Peters, M. \& Pedersen, K. (1978). Incidence of left-handers with inverted and noninverted writing position in a population of 5910 elementary school children. Neuropsychologia, 16, 743746.

Pyle, W. H. \& Drouin, A. (1932). Left-handedness: An experimental and statistical study. School and Society, 36, 253-256.

Reed, G.F. \& Smith, A.C. (1961). Laterality and directional preferences in a simple perceptual-motor task. Quarterly Journal of Experimental Psychology, 13, 122-124.

Searleman, A.; Porac, C. \& Coren, S. (1982). The relationship between birth stress and writing hand posture. Brain and Cognition, 1, 158-164.
Schwartz, M. (1990). Left-handedness and prenatal complications. Em S. Coren (Org.), Left-handedness: Behavioral implications and anomalies (pp. 75-97). Amsterdam: Elsevier.

Selzer, C. A. (1933). Lateral dominance and visual fusion. Harvard Monographs in Education, No. 12. Cambridge, Mass.: Harvard University Press.

Shepherd, M. (1986). The left-handed calligrapher. London: Thorsons Pub. Group.

Smith, A.C. \& Reed, G.F. (1959). An experimental investigation of the relative speeds of left and right-handed writers. Journal of Genetic Psychology, 94, 67-76.

Suen, C. Y. (1983). Handwriting generation, perception, and recognition. Acta Psychologica, 54, 295-312.

Szeligo, F.; Brazier, B. \& Houston, J. (2003). Adaptations of writing posture in response to task demands for left- and righthanders. Laterality, 8, 261-276.

Ure, D. (1969). Spelling performance of left-handed schoolchildren as affected by the use of a pencil modified to increase visual feedback. Journal of Experimental Child Psychology, 7 , 220-230.

Ziviani, J. (1984). Some elaborations on handwriting speed in 7 to - 14 year olds. Perceptual and Motor Skills, 58, 535-539.

Enviado: 17.03.2004 Revisado: 11.05.2004 Aceito: 20.05 .2004

\section{Sobre os autores:}

Francisca Morais da Silveira: Doutoranda em Psicologia: Teoria e Pesquisa do Comportamento Endereço para correspondência: Dept. de Psicologia da Universidade Federal do Maranhão São Luiz, Maranhão 65080-040 - E-mail: francmor@elo.com.br

William Lee Berdel Martin: PhD em Psicologia

Endereço para correspondência: Dept. de Psicologia Experimental da Universidade Federal do Pará Belém, Pará 66075-110 - E-mail: wlbm@cpgp.ufpa.br 